\title{
Dynamic Collision-Induced Dissociation (DCID) in a Quadrupole Ion Trap Using a Two-Frequency Excitation Waveform: II. Effects of Frequency Spacing and Scan Rate
}

\author{
Ünige A. Laskay, ${ }^{a}$ Olivier L. Collin, ${ }^{a}$ Jennifer J. Hyland, ${ }^{a, *}$ Brad Nichol, ${ }^{a}$ \\ Glen P. Jackson, ${ }^{a}$ Sofie P. Pasilis, ${ }^{b}$ and Douglas C. Duckworth ${ }^{c}$ \\ ${ }^{a}$ Center for Intelligent Chemical Instrumentation, Department of Chemistry and Biochemistry, Ohio \\ University, Athens, Ohio, USA \\ ${ }^{\mathrm{b}}$ Chemical Sciences Division, Oak Ridge National Laboratory, Oak Ridge, Tennessee, USA \\ c Pacific Northwest National Laboratory, Richland, Washington, USA
}

\begin{abstract}
Dynamic CID of selected precursor ions is achieved by the application of a two-frequency excitation waveform to the end-cap electrodes during the mass instability scan of a quadrupole ion trap (QIT) mass spectrometer. This new method permits a shorter scanning time when compared with conventional on-resonance CID. When the excitation waveform consists of two closely-spaced frequencies, the relative phase-relationship of the two frequencies plays a critical role in the fragmentation dynamics. However, at wider frequency spacings (>10 kHz), these phase effects are diminished, while maintaining the efficacy of closely-spaced excitation frequencies. The fragmentation efficiencies and energetics of $n$-butylbenzene and tetra-alanine are studied under different experimental conditions and the results are compared at various scan rate parameters between 0.1 and $1.0 \mathrm{~ms} / \mathrm{Th}$. Although faster scan rates reduce the analysis time, the maximum observed fragmentation efficiencies rarely exceed $30 \%$, compared with values in excess of $50 \%$ achieved at slower scan rates. The internal energies calculated from the simulations of $n$-butylbenzene at fast scan rates are $\sim 4 \mathrm{eV}$ for most experimental conditions, while at slow scan rates, internal energies above $5.5 \mathrm{eV}$ are observed for a wide range of conditions. Extensive ITSIM simulations support the observation that slowing the scan rate has a similar effect on fragmentation as widening the frequency spacing between the two excitation frequencies. Both approaches generally enhance CID efficiencies and make fragmentation less dependent upon the relative phase angle between the excitation waveform and the ion motion. (J Am Soc Mass Spectrom 2007, 18, 2017-2025) (c) 2007 American Society for Mass Spectrometry
\end{abstract}

$\mathrm{T}$ The use of quadrupole ion traps (QITs) as a platform to perform tandem mass spectrometry was made possible largely by the development of resonance excitation by Louris et al. [1]. In this approach, a supplementary AC voltage is applied to the end-cap electrodes as the ions are held at a fixed trapping potential. If the applied excitation frequency matches the secular frequency of the precursor ion, the on-resonance condition results in an increase of the ion kinetic energy. Energetic collisions with the bath gas then occur and the kinetic energy of the ions is converted to internal energy. This, in turn, leads to cleavage of the chemical bonds and fragmentation takes place. One of the drawbacks of applying this method is the resonance ejection of the ions when, due to the in-

Address reprint requests to Dr. Glen P. Jackson, Department of Chemistry and Biochemistry, Ohio University, 136 Clippinger Laboratories, Athens, OH 45701-2979, USA. E-mail: jacksong@ohio.edu

* Current address: Department of Chemistry, University of Illinois, Urbana, Illinois. creased kinetic energy, their motion becomes unstable and the ions are ejected without being mass analyzed [2]. Although it is quite straightforward to calculate the approximate secular frequency of an ion under a specific set of experimental conditions, the only way to ensure on-resonance excitation is through lengthy empirical calibration procedures. Consequently, there has been an intense search to find new, improved ways to perform and optimize tandem mass spectrometry in QITs.

A widely used approach to improve the performance of the QITs is the application of custom-tailored waveforms to the end-cap electrodes. Goeringer and coworkers tackled the problem of selective ion accumulation by applying filtered noise fields (FNF) [3], while Doroshenko and Cotter described the means of injecting ions in an increasing $\mathrm{rf}$ field to improve trapping efficiency [4, 5]. Fulford et al. used a low-frequency rf signal coupled to the end-cap electrodes in a monopolar fashion to achieve resonance ejection of selected ions [6]; Lammert 
et al. proposed the application of a short dc pulse to activate the precursor ion [7]; Murrell et al. developed a method for fast excitation by applying a high amplitude excitation frequency [8]; Paradisi et al. modified the scan function by introducing two excitation periods separated by a cooling period [9]; and, recently, the Glish research group reported the benefits of using a high-amplitude short-time excitation (HASTE) to recapture low-mass product ions [10]. Most of these methods still require resonance tuning or other optimization of experimental parameters to attain satisfactory CID efficiencies.

DCID is a new fragmentation method that may be achieved in most commercial instruments without any hardware modifications and allows for fast and quite efficient fragmentation of selected precursor ions [11]. Single-frequency DCID has been successfully applied for the fragmentation of biomolecules such as leucine enkephalin, insulin chain A and substance P [12]. Previous studies have focused on characterizing the fragmentation energetics and efficiencies when a single frequency is applied to the end-cap electrodes during mass acquisition [13], and understanding the waveform-ion interaction for two-frequency DCID [14]. The effect of excitation amplitude and $\mathrm{q}_{\mathrm{z}}$ has been described in a companion paper to this [14]; the effect of frequency spacing and mass scanning rate are presented here.

\section{Experimental}

For the analysis of gaseous samples such as n-butylbenzene, the experimental data were collected with a Thermo Finnigan Polaris Q quadrupole ion trap mass spectrometer (Austin, TX) as previously described [13]. The ESI experiments using tetra-alanine were conducted on a Thermo Finnigan Polaris Q mass spectrometer modified with an electrospray interface described earlier [15]. Tetra-alanine ( $>99 \%)$ purchased from Bachem (King of Prussia, PA) was dissolved in a 1:1 (vol:vol) methanol:water mixture containing $0.1 \%$ formic acid (Fluka, $78 \%$, Buchs, Switzerland) to a 0.45 to $0.48 \mathrm{mM}$ concentration. Spray voltage was typically 4 to $4.5 \mathrm{kV}$; solvent flow rate was set to 3.5 to $4 \mu \mathrm{L} / \mathrm{min}$.

The two-dimensional contour plots presented in this report were constructed in Origin (OriginLab, Northampton, MA). Each plot shows the results of more than 18,000 different experimental conditions in a sequence-looped format [14]. The ability to perform experiments in such vast number is offered by the flexible programming permitted by the Visual Basic platform. Because the primary focus of our present study is the understanding of the effects of each parameter involved in our experiments, the construction of these contour plots allow for a direct comparison of the energetics under the different investigated conditions.

A two-frequency excitation waveform is applied to the end-cap electrodes in a dipolar fashion during mass acquisition; the amplitude of excitation refers to the amplitude $\left(\mathrm{V}_{p-p}\right)$ of the excitation waveform applied between the end-cap electrodes. The phase angle term describes the initial relative phase relationship of the two excitation frequencies at the onset of the mass acquisition scan and does not suppose a known phaserelationship to the $\mathrm{rf}$ frequency on the ring electrode during resonance conditions or to the ion motion during resonance conditions. The difference between the two frequency values is referred to as frequency spacing and the $\mathrm{q}_{\mathrm{z}}$ value is calculated from the first (smaller) of the two applied excitation frequencies. The default scanning rate of $0.18 \mathrm{~ms} / \mathrm{Th}$ was used for all experiments, unless otherwise stated.

The internal energy deposited by an arbitrary excitation waveform may be approximated by the use of thermometer molecules, such as $n$-butylbenzene. These species have well-known fragmentation energetics, as, depending on the deposited internal energy, fragmentation may occur through two entirely different mechanisms. At low internal energies, the direct cleavage product is more abundant $(m / z 92)$, while higher internal energy depositions lead to an intramolecular rearrangement; in this case the most abundant product ion has an $\mathrm{m} / \mathrm{z}$ of 91 . With the aid of a calibration curve correlating the fragment ion ratios and total ion internal energy [16], the fragmentation energetics may be approximated for each experimental condition in part.

The fragmentation efficiency herein will be calculated as the abundance of the 91,92, 105, and 119 Th fragment ions relative to the intensity of the isolated precursor ion.

\section{Results and Discussion}

To better understand the following experimental results, it is helpful to create a physical vision of the excitation process using the properties of a sum-of-sines waveform [12]. Although this interpretation is a somewhat oversimplified version of the "true" excitation mechanism, it aids in the understanding and description of the general trends observed. Consider the example when two frequencies are applied to the end-cap electrodes in a dipolar fashion: the resulting waveform will have a beat-pattern where the position of the nodes and crests is trigonometrically equivalent to a waveform of $\operatorname{COS}\left(\left\{\omega_{1}+\omega_{2}\right\} t / 2\right)$, modulated by $\operatorname{SIN}\left(\left\{\omega_{1}-\right.\right.$ $\left.\left.\omega_{2}\right\} t / 2\right)$. The modulation frequency defines the nodes (minima) and crests (maxima) in the time domain of the interference pattern. At close frequency spacings, the $\operatorname{SIN}\left(\left\{\omega_{1}-\omega_{2}\right\} t / 2\right)$ term will be small, thus a "slow beat" is observed in the time domain. When the two frequencies are applied further apart, the term will be larger, indicating a faster beat pattern in the time domain of the interference pattern of the excitation waveform. It was previously shown that the fragmentation of the selected precursor ions depends on the time at which they come into resonance with the interference pattern of the excitation waveform, and on the excitation amplitude [14]. When the excitation waveform goes through a minimum, or a node, at the time of excitation, reduced 

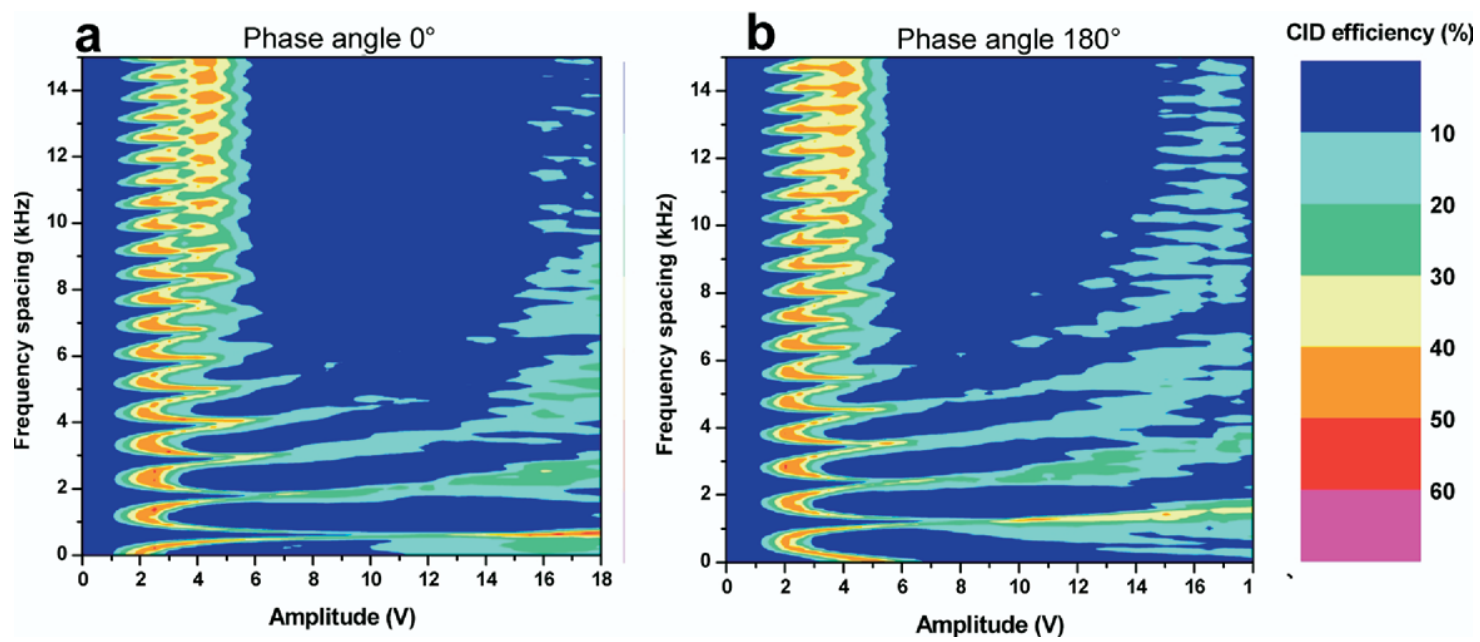

Figure 1. CID efficiencies of $n$-butylbenzene achieved by application of a two-frequency excitation waveform when the first of the two frequencies is held at $171 \mathrm{kHz}$ and the second frequency is gradually changed between experiments by increments of $0.02 \mathrm{kHz}$ from 171 to $186 \mathrm{kHz}$, at excitation waveform amplitudes between 0 and $18 \mathrm{~V}$. The relative phase angle between the two frequencies is (a) $0^{\circ}$ and (b) $180^{\circ}$

kinetic energy deposition is expected and the fragmentation is less efficient. Conversely, when the resonance condition occurs at a maximum or the crest of the waveform, more kinetic energy is deposited and the fragmentation is more efficient. At large excitation amplitudes, additional and more complex phenomena are observed, mainly due to off-resonance excitation, as will be demonstrated.

Figure 1a shows the experimentally determined fragmentation efficiencies of $n$-butylbenzene at more than 27,500 different experimental conditions obtained in a single sequence-looped experiment. The first of the two frequencies was held constant at $171 \mathrm{kHz}$ and the second frequency was incrementally changed by 0.02
$\mathrm{kHz}$ intervals from 171 to $186 \mathrm{kHz}$. Excitation amplitudes between 0 and $18 \mathrm{~V}_{p-p}$ in $0.5 \mathrm{~V}_{p-p}$ increments were tested for each of the frequency spacings. The relative phase angle between the two excitation frequencies was $0^{\circ}$ for all experiments shown in Figure 1a and set to $180^{\circ}$ for those in Figure 2b. Several common features may be observed when comparing the two plots shown in Figure 1. It is immediately apparent that, regardless of the relative phase angle or frequency spacing of the two excitation frequencies, excitation amplitudes below $1 \mathrm{~V}_{p-p}$ do not cause significant fragmentation. It can also be noted that at each frequency spacing, there are several amplitude intervals that allow for fragmentation efficiencies larger than $50 \%$. When the
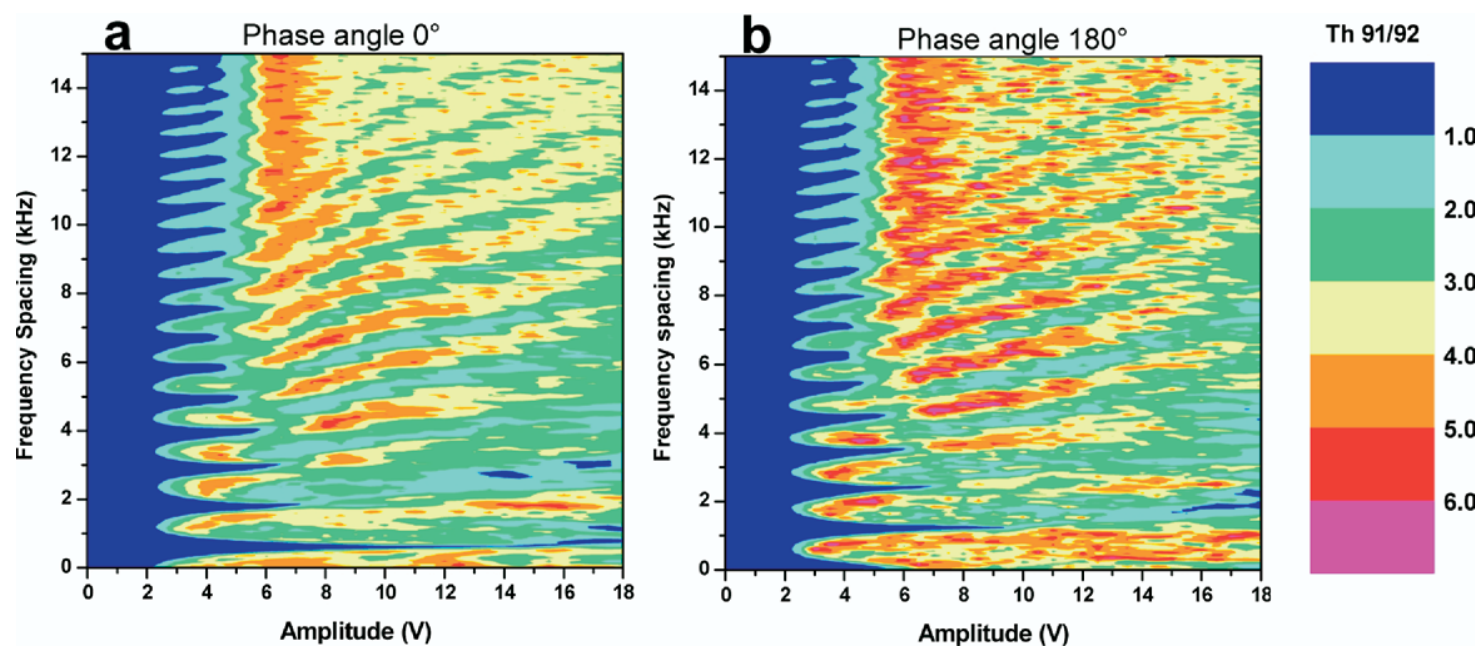

Figure 2. $n$-Butylbenzene fragment ion ratios of $91 / 92$ Th fragment ion ratios achieved by application of a two-frequency excitation waveform when the first of the two frequencies is held at 171 $\mathrm{kHz}$ and the second frequency is gradually changed between experiments by increments of $0.02 \mathrm{kHz}$ from 171 to $186 \mathrm{kHz}$; at excitation amplitudes between 0 and $18 \mathrm{~V}$. The relative phase angle between the two frequencies is (a) $0^{\circ}$ and (b) $180^{\circ}$. 
excitation amplitude is above $5.5 \mathrm{~V}_{p-p}$ the kinetic energy of the isolated precursor ions is increased significantly, causing the ejection of ions without significant fragmentation.

The differences between the two plots shown in Figure 1 are most apparent in the frequency-spacing domain. To facilitate the understanding of these differences, let us consider the situation when the twofrequency excitation waveform is applied at a $2 \mathrm{~V}_{p-p}$ amplitude and a relative phase angle of the two excitation frequencies set to $0^{\circ}$ (Figure 1a). At frequency spacings between 0 and $0.2 \mathrm{kHz}$, the two excitation frequencies constructively interfere during on-resonance conditions, so the ions gain considerable kinetic energy during their short interaction time with the excitation waveform. For these conditions, fragmentation efficiencies above $50 \%$ may be observed. By increasing the frequency spacing from 0.2 to $1 \mathrm{kHz}$, the CID efficiency is decreased because the resonance conditions occur during a node in the interference pattern of the excitation waveform. A further increase in the frequency spacing allows for efficient fragmentation, followed by yet another minimum. This trend may be followed for the entire frequency spacing domain, alternating between efficient and inefficient fragmentation depending on the interference pattern of the excitation waveform during resonance conditions. A similar pattern is observed when the two excitation frequencies are applied at the same amplitude, except at an initial relative phase angle of $180^{\circ}$. In Figure $1 \mathrm{~b}$, the positions of the maxima and minima are shifted in the frequency domain in a systematic manner. In short, changing the frequency spacing has a somewhat similar effect as shifting the relative positions of the crests and nodes of the interference pattern in the time domain.

A region of great interest in Figure 1 is where changes in the frequency spacing or phase angle relationship do not greatly affect the fragmentation efficiencies. At frequency spacings above $10 \mathrm{kHz}$ and excitation amplitudes between 4 and $5 \mathrm{~V}_{p-p}$, the fragmentation efficiencies of $n$-butylbenzene are between $30 \%$ and $50 \%$ regardless of the relative phase angle of the two frequencies. In this case, the amplitude of the excitation waveform is presumably large enough to cause offresonance excitation so that regardless of the phase angle of the two excitation frequencies, sufficient kinetic energy is deposited into the precursor ion to cause relatively efficient fragmentation. Another potential factor is that as the frequency spacing between the two excitation frequencies increases, the beat frequency in the interference pattern also increases. This implies that the precursor ion will pass through multiple nodes and crests in the interference pattern, even for the short duration for which they are resonant, and will likely absorb a similar amount of power regardless of the phase angle.

Figure 2 shows the experimentally determined 91/92 fragment ion ratios for $n$-butylbenzene for the same data used to create Figure 1. In general, highly energetic fragmentations do not correlate with efficient DCID conditions. A notable trend is that excitation amplitudes below $4.5 \mathrm{~V}_{p-p}$ allow for high internal energy depositions only when the frequency spacing is less than 6 $\mathrm{kHz}$; however, excitation amplitudes above $6 \mathrm{~V}_{p-p}$ can provide energetic conditions at most frequency spacings, especially at those greater than $6 \mathrm{kHz}$. The fact that larger excitation amplitudes are required for energetic fragmentation at large frequency spacings can be reasoned through two possible explanations: (1) that the energy absorbed by the ions from the two excitation frequencies is more uncoupled, meaning that the ions are excited by one frequency, independently of the other. The ions then pick up kinetic energy from the second frequency later during the scan when they pass through resonance conditions with the second frequency. (2) Wide frequency spacings produce an interference pattern with more frequent nodes and crests in the time domain. Therefore, during the period of resonance excitation, the ions will experience multiple nodes and crests and will absorb a certain average amount of power from the waveform. The absorbed power will be similar whether the resonance conditions start at a crest or a node, which is why the fragmentation patterns change less at wider frequency spacings.

Additional frequency-spacing experiments were run on singly protonated tetra-alanine (see Supplementary Material section, which can be found in the electronic version of this article) to test the success of DCID for fragmentation of small biomolecules. It was found that at frequency spacings larger than $12 \mathrm{kHz}$ the resonance ejection is diminished. The spectra observed for large $(>20 \mathrm{kHz})$ frequency spacings displayed no significant changes relative to spectra observed at $20 \mathrm{kHz}$. This observation, as well as the fact that resonance ejection is diminished at high-frequency spacings can again be reasoned through decoupling of the excitation frequencies, or through considering the interference pattern of the excitation waveform. Consequently, the amount of internal energy deposited is on average smaller than for more closely spaced frequencies and less sensitive to any changes in the frequency spacing.

In the next series of experiments, the effects of frequency spacing and relative phase angle on $n$-butylbenzene fragmentation were studied at three different excitation amplitudes. For all experiments shown in Figure 3a the amplitude of the excitation waveform was held at $2 \mathrm{~V}$. The plot shows diagonal ridges of optimum CID efficiencies. The physical model describing the excitation period with the aid of the shape of the sum-of-sines waveform works well at close frequency spacings. Here, a change in the relative phase angle of the two excitation frequencies causes an apparent shift in the position of nodes and crests; thus, the fragmentation energetics changes according to whether the ions come on-resonance during a node or crest in the beat pattern. A change in the frequency spacing, while keeping the phase angle constant, causes similar effects: the position of the nodes and crests of the excitation waveform will 

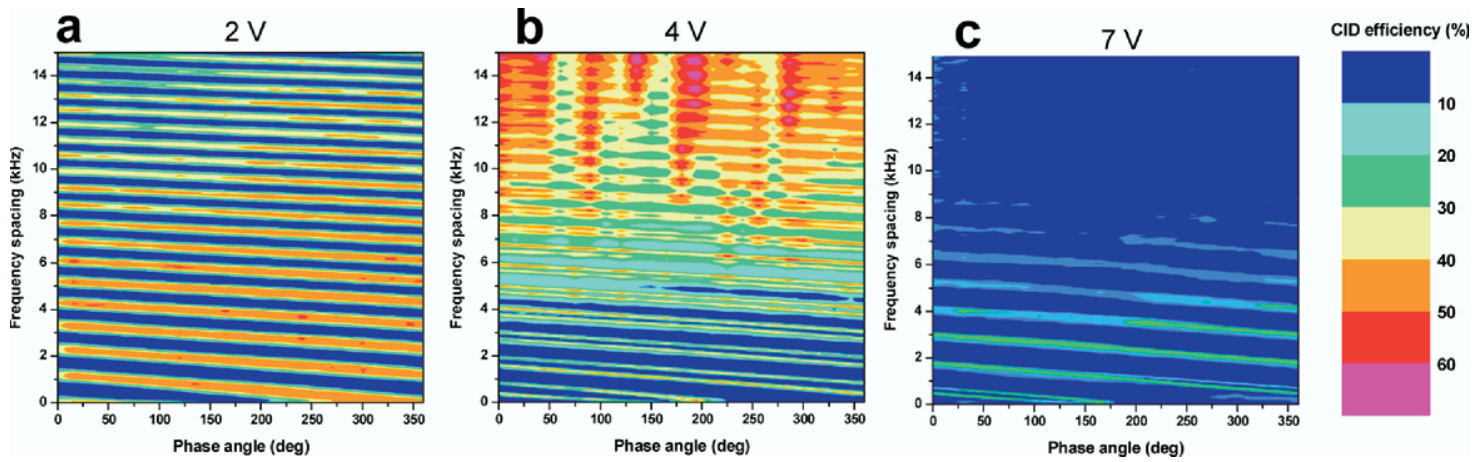

Figure 3. CID efficiencies for $n$-butylbenzene achieved by application of a two-frequency excitation waveform when the first of the two frequencies is held at $171 \mathrm{kHz}$ and the second frequency is gradually changed between experiments by increments of $0.02 \mathrm{kHz}$ from 171 to $186 \mathrm{kHz}$; at relative phase angles of the two frequencies between $0^{\circ}$ and $360^{\circ}$. The amplitude of the excitation waveform is (a) $2 \mathrm{~V}$; (b) $4 \mathrm{~V}$; (c) $7 \mathrm{~V}$.

shift as the frequency spacing increases. At wide frequency spacings the physical model cannot be applied as rigorously, as shown by the gradual loss of features at wider frequency spacings in Figure $4 \mathrm{~b}$ and $\mathrm{c}$.

Figure $3 \mathrm{~b}$ and $\mathrm{c}$ show the CID efficiencies when the amplitude of the excitation frequency is 4 and $7 V_{p-p}$, respectively. At an excitation amplitude of $4 \mathrm{~V}_{p-p}$, the CID efficiencies at frequency spacings greater than 10 $\mathrm{kHz}$ are much less sensitive to the relative phase angle than at $2 \mathrm{~V}_{p-p}$ excitation amplitude, and relatively efficient fragmentation is possible at almost any given phase angle. Applying excitation waveforms at $7 \mathrm{~V}_{p-p}$ results in a loss of fragmentation efficiency due to an increase in resonance ejection of the isolated precursorion.

The 91/92 fragment ion ratios calculated for the same set of experiments are shown in Figure 4. Excitation amplitudes of $2 \mathrm{~V}_{p-p}$ (shown in Figure 4a) result in low fragmentation energetics for all frequency spacings and relative phase angles studied. More energetic conditions may be achieved at closely spaced excitation frequencies when the excitation amplitude is increased to $4 \mathrm{~V}_{p-p}$, as shown in Figure $4 \mathrm{~b}$. By increasing the excitation amplitude to $7 \mathrm{~V}_{p-p}$, highly energetic frag- mentations of $n$-butylbenzene is possible even at large frequency spacings, as shown in Figure 4c. The results shown in these plots support the above formulated assumption that the phase-angle does not strongly influence fragmentation efficiencies or energetics when the frequency spacing between the two excitation frequencies is $>10 \mathrm{kHz}$.

These results indicate that a competition between efficient and energetic fragmentation condition exists at all experimental settings studied. Given the complexity of two-frequency DCID, the extensive number of parameters that require optimization might appear difficult; however, selection of wide frequency spacings $(>10 \mathrm{kHz})$ between two components of a two-frequency DCID waveform serves to reduce the complexity of optimization.

Simulations using ITSIM 5.0 were conducted to model the fragmentation energetics of $n$-butylbenzene at different relative phase angles and frequency spacings of the two excitation frequencies at an excitation amplitude of $2 \mathrm{~V}_{p-p}$. The variables of the experiment were previously described [14]. The simulations were conducted for frequency spacings between 171 and
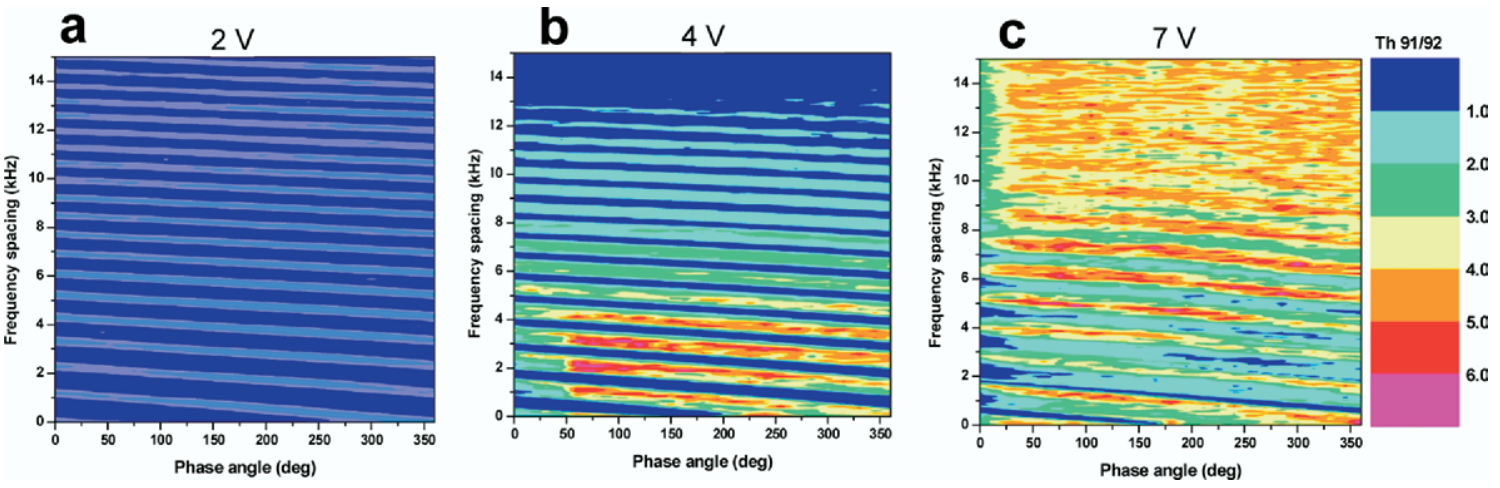

Figure 4. n-Butylbenzene fragment ion ratios of Th 91/92 achieved by application of a two-frequency excitation waveform when the first of the two frequencies is held at $171 \mathrm{kHz}$ and the second frequency is gradually changed between experiments by increments of $0.02 \mathrm{kHz}$ from 171 to $186 \mathrm{kHz}$; at relative phase angles of the two frequencies between $0^{\circ}$ and $360^{\circ}$. The amplitude of the excitation waveform is (a) $2 \mathrm{~V}$; (b) $4 \mathrm{~V}$; (c) $7 \mathrm{~V}$. 
$176 \mathrm{kHz}$ in increments of $0.05 \mathrm{kHz}$ and at phase angles from $0^{\circ}$ to $360^{\circ}$ by increments of $20^{\circ}$ for each frequency spacing. This translates into a total of 1881 different simulation experiments to provide confirmation for the experimental data. Figure 5a shows the total amplitude of the excitation waveform at precisely $5.13 \mathrm{~ms}$ at different frequency spacings and phase angles. The mark at $5.13 \mathrm{~ms}$ is the theoretical moment when the ion is on-resonance with the smaller of the two frequencies of the DCID waveform $(171 \mathrm{kHz})$. Figure $5 \mathrm{~b}$ shows the calculated internal energy of $n$-butylbenzene precursor ions from the ITSIM 5.0 simulations. Figure $5 \mathrm{c}$ shows the experimentally determined relative internal energies measured by the ratio of the product ions 91/92. The diagonal lines with negative slopes in all three plots demonstrate a good qualitative agreement between the three parameters, and a clear phase- and frequencyspacing dependence of the deposited internal energy. However, the simulations overestimate the number of the observed maxima and minima in the internal energy of the ions, indicating a more rigid correlation between the interference pattern of the excitation waveform at a given instant and the amount of deposited internal energy. Close examination of the internal energy obtained by theoretical and experimental results for the different excitation waveforms (Figure $5 b$ and c) reveals that the slopes of the lines indicating maxima in the internal energies are quite different. The simulation results follow the trend of the excitation amplitude calculated in Figure 5a, whereas the less frequent ridges in the experimental data suggest that the simulations could be underestimating the importance of some other factors, such as off-resonance excitation/de-excitation. These findings indicate that the sum-of-sines model offers a reasonable explanation in describing most of the phenomena involved in DCID, especially at close $(<5 \mathrm{kHz})$ frequency spacings. However, the exact process of internal energy deposition is a more complex process that cannot be described merely by the interference pattern of the excitation waveform at the theoretical instant of on-resonance excitation. In addition to the shape of the excitation waveform, internal energy deposition is dependent on a variety of experimental parameters, such as parent ion $m / z$, nature and pressure of bath gas, amplitude of the excitation waveform during on-resonance conditions, total duration of excitation, and time allotted for cooling of the excited species. Therefore, it is hypothesized that the differences between hypothetical and observed internal energies are the result of the added effect of all these parameters.

The effects of mass acquisition scan rate on DCID fragmentation efficiency was also investigated under different two-frequency DCID conditions. Sequencelooped experiments were conducted wherein the twofrequency excitation waveform was applied at $\mathrm{q}_{\mathrm{z}} 0.45$ $(171,172 \mathrm{kHz})$ with amplitudes varying between 0 and $18 \mathrm{~V}_{p-p}$ and phase angles between $0^{\circ}$ and $360^{\circ}$. This set of experiments was performed at scan rate parameters of $0.10,0.18,0.36,0.50,0.72$, and $1.00 \mathrm{~ms} / \mathrm{Th}$. The DCID
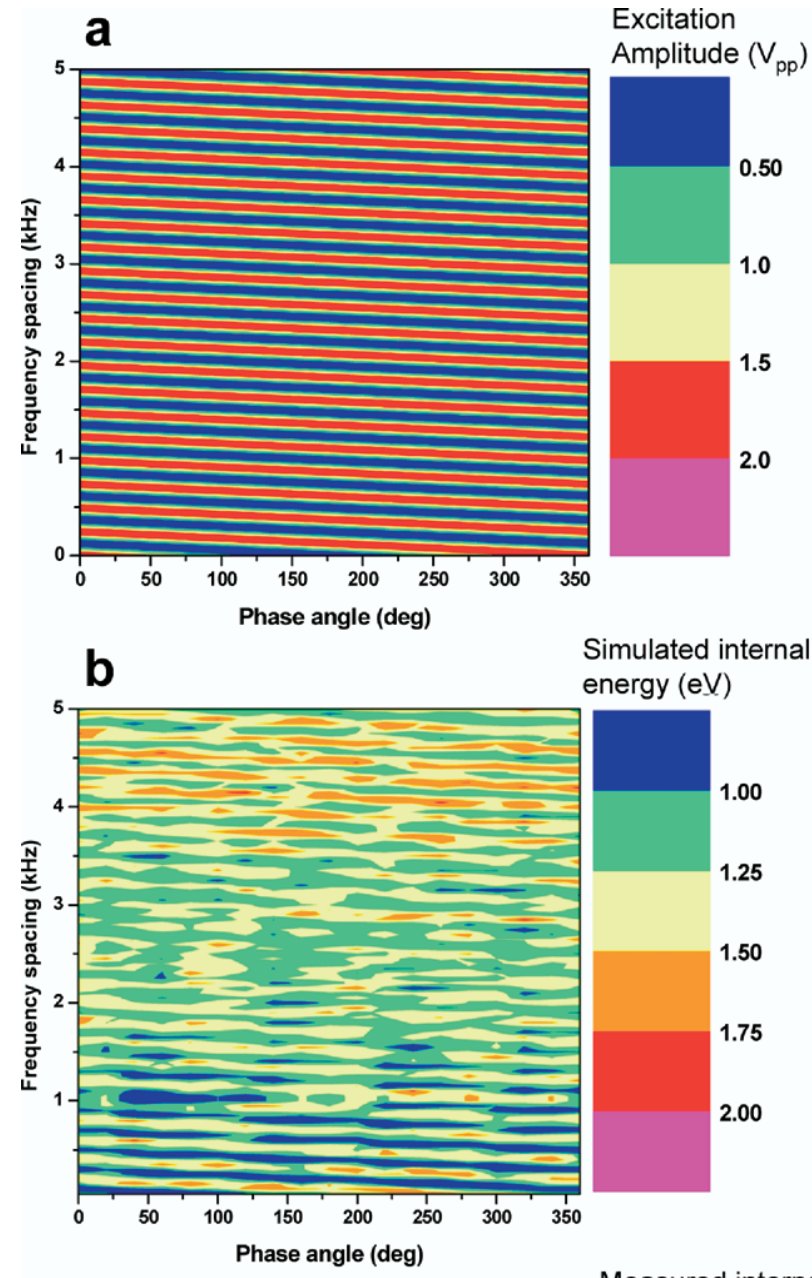

Simulated internal energy $(\mathrm{eV})$

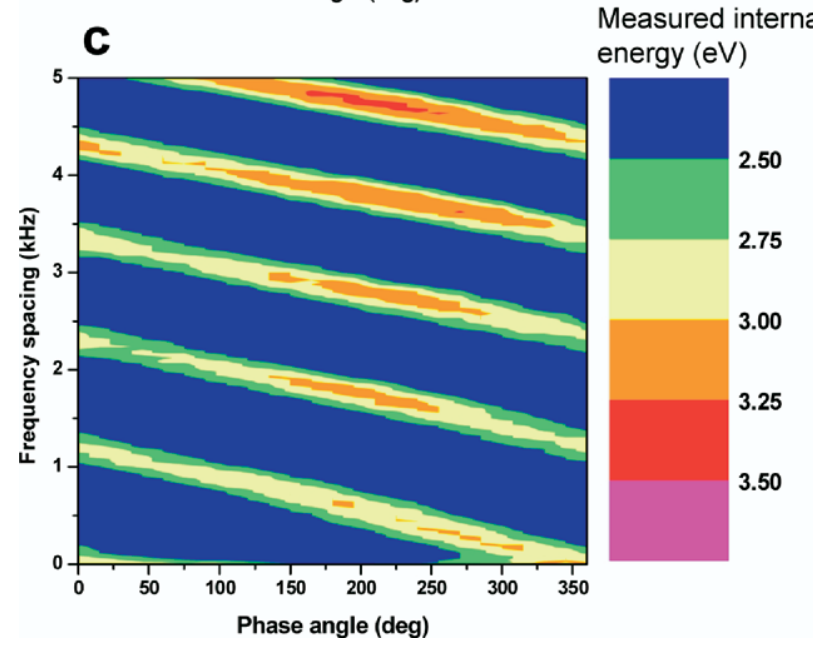

Figure 5. Two-frequency DCID excitation waveform of 171 and $171+0.02 \mathrm{n}(n=0-250)$ of relative phase angle $0^{\circ}-360^{\circ}$ and excitation amplitude $2 \mathrm{~V}_{p-p}$ : (a) amplitude of a sum-of-sines waveform at $5.13 \mathrm{~ms}$; (b) simulated internal energy of $n$-butylbenzene in the ion trap $9 \mathrm{~ms}$ after resonance excitation; (c) experimentally determined internal energy of $n$-butylbenzene.

efficiencies obtained for this set of experiments are shown in Figure 6.

The scan rate has a major influence on CID efficiencies when a two-frequency excitation waveform is used 

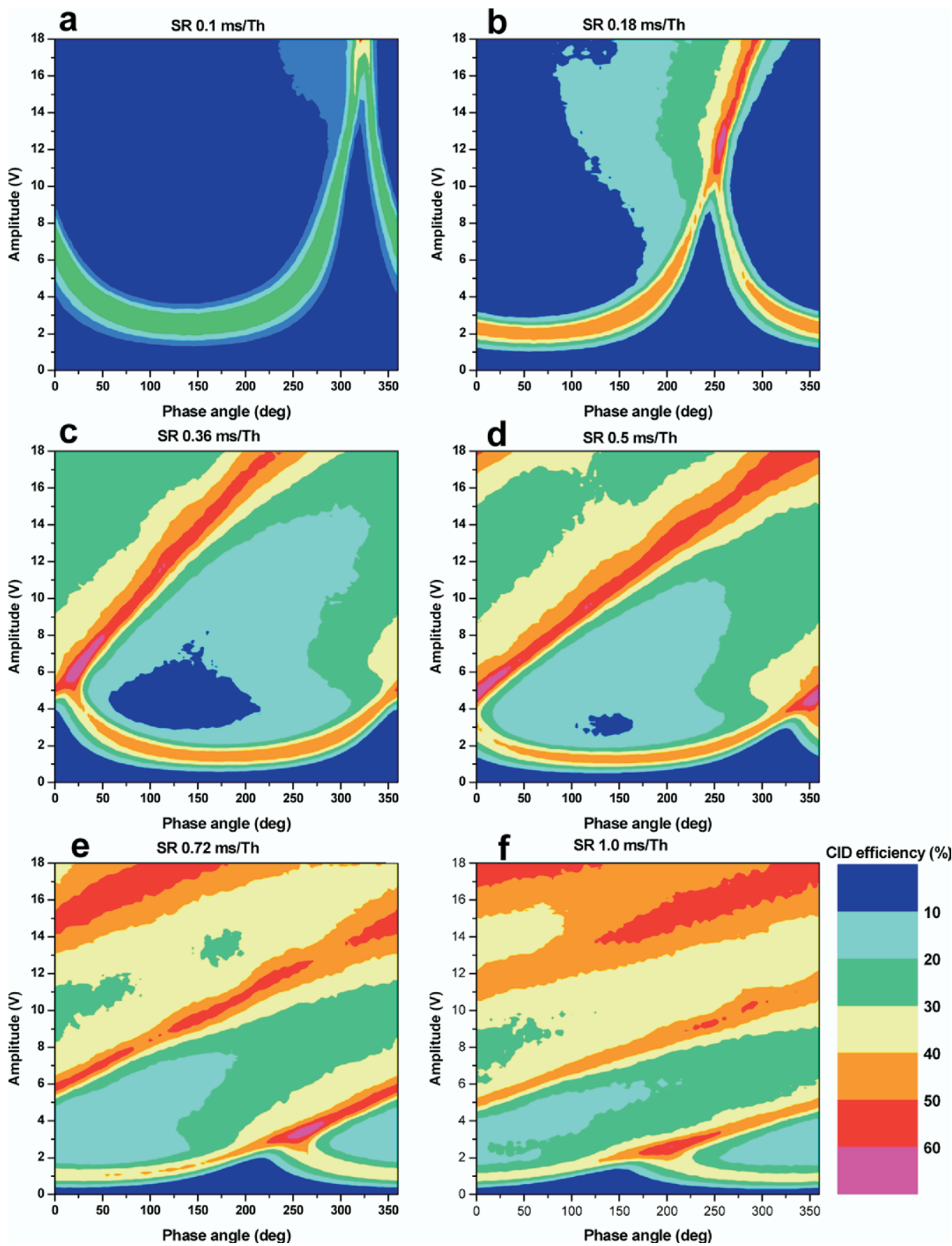

Figure 6. CID efficiencies of $n$-butylbenzene at different excitation amplitudes and relative phase angles of the two excitation frequencies $(171,172 \mathrm{kHz})$, when the scan rate parameter is a) $0.1 \mathrm{~ms} / \mathrm{Th}$, (b) $0.18 \mathrm{~ms} / \mathrm{Th},(\mathbf{c}) 0.36 \mathrm{~ms} / \mathrm{Th}$, (d) $0.5 \mathrm{~ms} / \mathrm{Th},(\mathbf{e}) 0.72 \mathrm{~ms} / \mathrm{Th}$, and (f) $1.0 \mathrm{~ms} / \mathrm{Th}$.

to fragment the selected precursor ion. The scan rate is very closely correlated with the time period a certain ion is in resonance with the excitation waveform: ions are resonant with the excitation frequency for a shorter period at faster scan rates than at slower scan rates. This is similar to the concept of fluence, first described by March et al. [17] with respect to conventional CID. This model purports that the fragmentation efficiency is correlated with the product of the excitation amplitude and the duration of the excitation. Consequently, the maximum fragmentation efficiencies shown in Figure 6a calculated for a scan rate parameter of $0.1 \mathrm{~ms} / \mathrm{Th}$ are at best $\sim 30 \%$, while at slower scan rates (Figure 6c-f) CID efficiencies in excess of $60 \%$ are possible. When the scan rate parameter is $0.1 \mathrm{~ms} / \mathrm{Th}$ (Figure 6a), the maximum CID efficiency values are arranged along a " $u$ "-shaped curve in such a way that at each phase angle a single, well-defined excitation amplitude is required to achieve fragmentation. At the default scan rate parameter of $0.18 \mathrm{~ms} / \mathrm{Th}$ (shown in Figure 6b), a phase-dependence is 
still observable for excitation amplitudes below $5 \mathrm{~V}$; however, the appearance of a larger area at high excitation amplitudes, where noticeable fragmentation efficiencies are achieved, suggests that off-resonance excitation might be occurring. At large excitation amplitudes, the relative phase angle of the two excitation frequencies is less critical, as long as the excitation takes place near the crest of the excitation waveform. This trend is clearly more accentuated at slower scan rates, as shown in Figure $6 c-f$, to the point where the phase-dependence is diminished even at low excitation amplitudes (Figure 6f).

Figure 2S (Supplementary Material) shows the fragmentation of singly protonated tetra-alanine investigated under different DCID conditions. When the excitation waveform consists of two frequencies spaced 20 $\mathrm{kHz}$ apart (83 and $103 \mathrm{kHz}$ ), the beat in the interference pattern of the excitation waveform is much faster in the time domain. Consequently, even at fast scanning rates, tetra-alanine is more likely to meet a crest (or multiple crests) in the excitation waveform during on-resonance conditions, thus the fragmentation energetics are much improved. Similar to the findings of the study conducted on $n$-butylbenzene, slower scan rates cause more energetic interactions between the precursor ion and the excitation waveform, leading to the resonance ejection of precursor ions.

It is shown that for close frequency spacings the relative phase-dependence of the fragmentation efficiencies and energetics is more accentuated at fast scan rates. Here, the ions will only interact with the excitation waveform for a short period that corresponds to a node or crest of the interference pattern of the excitation waveform, so the absorbed power is very sensitive to the shape of the waveform-i.e., the relative phase angle of the two excitation frequencies. At slow scan rates, ions are on-resonance with the excitation waveform for a longer time, so the total power absorbed by the ions during excitation will be increased. According to the sum-of-sines analogy, the interference pattern will go through multiple beats during excitation of the ions during slow scanning DCID, so the ions will gain a similar amount of power regardless of the initial phase angle with which they come into resonance with the waveform. Another way to picture the slow scanning DCID is by assuming that the ions pick up kinetic energy from the two excitation frequencies at two discrete periods, and that the time between the two periods is so long in the slow scanning DCID experiment that the ions will lose any phase "memory" from the first excitation frequency by the time they come on-resonance with the second frequency.

\section{Summary of Single- and Two-Frequency DCID}

We have presented the effect of several experimental parameters, such as excitation amplitude and frequency [14] and frequency spacing and scan rate (present report) on the fragmentation energetics and efficiencies when a one- $[12,13]$ or two-frequency excitation waveform is used to fragment selected precursor ions during mass acquisition. It is evident that several other parameters influence the energy deposition process (bath gas pressure, space charge effects) and these parameters will require future investigation. Several interdependent variables have been characterized with respect to fragmentation efficiency and energetics. The results are summarized as follows: increasing the scan speed reduces precursor ion excitation time and requires increased DCID excitation amplitude to achieve the same fragmentation efficiency. Increased excitation amplitude (1) may increase off-resonance effects, and (2) may result in more resonant frequency ejection while reducing the time between collisional activation, dissociation and mass-instability ejection. Increasing the scan speed therefore (1) may not allow sufficient time for fragment ions to form, (2) reduces cooling time of fragment ions before ejection, (3) may degrade scanning resolving power, (4) may increase S/N, (5) minimizes deleterious product ion secondary reaction times, and (6) reduces precursor ion ejection for a given resonant frequency amplitude. As with conventional, on-resonance CID, DCID waveforms consisting of smaller excitation frequencies result in fragmentation at smaller $q_{z}$ values, smaller Dehmelt pseudopotential well-depths, and may result in more resonance ejection, poorer CID efficiencies, but improvements in the ability to recapture low mass products ions. Conversely, fragmentation at larger excitation frequencies, larger $\mathrm{q}_{\mathrm{z}}$ values, and larger well depths tends to improve fragmentation efficiencies, but limits the ability to recapture low mass product ions.

Increasing from one to two resonant frequencies in DCID adds many additional variables: frequency spacing, amplitude of frequency 1, amplitude of frequency 2 , and relative phase angles of the two excitation frequencies. The effects of relative amplitudes other than 50:50 have yet to be studied in detail. Twofrequency DCID is therefore more complex to optimize than single-frequency DCID. However, there are benefits to two-frequency DCID. In general, larger internal energy depositions are possible using two-frequency DCID waveform than employing a single frequency. Increasing the frequency spacing between the two excitation frequencies simplifies tuning for optimal performance and reduces resonant ejection of precursor ions for a fixed excitation amplitude.

\section{Conclusions}

Dynamic CID is shown to be a viable alternative for on-resonance excitation for small organic molecules such as $n$-butylbenzene and small synthetic peptides such as tetra-analine. Two-frequency DCID allows for a large internal energy deposition and better fragmentation efficiencies than single frequency DCID, but the phase angle of the two frequencies must be optimized to assure the best possible fragmentation capabilities. 
The necessity for "phase tuning" may be overcome by two approaches: (1) using excitation frequency spacings larger than $10 \mathrm{kHz}$ (at a scan rate parameter of 0.18 $\mathrm{ms} / \mathrm{Th}$ ), and (2) using excitation frequency spacings closer than $10 \mathrm{kHz}$, but slowing down the mass acquisition scanning rate. The effects of the two variables are very similar; they both serve to increase number of nodes and crests that the ions are exposed to during the short period with which the ions can absorb energy from the excitation waveform. The observed result is that when the internal energy gained by the ions is some function of multiple beat patterns of an excitation waveform, that function will be less sensitive to the initial phase angle of the excitation waveform.

Why is reducing the phase dependency important? In an effort to utilize two-frequency DCID for on-line analyses of complex mixtures, the authors understand the need to find a "universal" excitation waveform that allows for efficient and/or energetic fragmentation of precursor ions of different $m / z$ values, without the need for phase optimization. It is hypothesized that generating a waveform consisting of two widely-spaced frequencies-or three or more frequencies-could circumvent the requirement for phase tuning. Such waveforms could offer a means for sequential fragmentation of several precursor ions (such as reducing polyatomic interference ions in ICP-QIT-MS) or for achieving an efficient, mass-independent, one-sizefits-all waveform for fragmenting precursor ions of any $m / z$. Although single-frequency DCID offers a more simplified approach, two-frequency DCID can offer better fragmentation efficiencies and larger internal energy depositions, if tuned appropriately.

\section{Acknowledgments}

The authors gratefully acknowledge financial support from NSF (grant number DBI 0649747). OLC is grateful for financial support from Fonds Québécois de la Recherche sur la Nature et les Technologies. SPP acknowledges an Oak Ridge National Laboratory (ORNL) appointment through the ORNL Postdoctoral Research Associates program. A portion of this work was carried out at ORNL and was sponsored by the Division of Chemical Sciences, Geosciences, and Biosciences, United States Department of Energy, Office of Basic Energy Sciences. ORNL is managed and operated by UT-Battelle, LLC, for the United States Department of Energy under contract DE-AC05-00OR22725.

\section{References}

1. Louris, J. N.; Cooks, R. G.; Syka, J. E. P.; Kelley, P. E.; Stafford, G. C.; Todd, J. F. J. Instrumentation, Applications, and Energy Deposition in Quadrupole Ion-Trap Tandem Mass Spectrometry. Anal. Chem. 1987, 59(13), 1677-1685.

2. Charles, M. J.; McLuckey, S. A.; Glish, G. L. Competition Between Resonance Ejection and Ion Dissociation During Resonance Excitation in a Quadrupole Ion Trap. J. Am. Soc. Mass Spectrom. 1994, 5, 1031-1041.

3. Goeringer, D. E.; Asano, K. G.; McLuckey, S. A.; Hoekman, D.; Stiller, S. W. Filtered Noise Field Signals for Mass-Selective Accumulation of Externally Formed Ions in a Quadrupole Ion Trap. Anal. Chem. 1994, 66, 313-318.

4. Doroshenko, V. M.; Cotter, R. J. Injection of Externally Generated Ions Into an Increasing Trapping Field of a Quadrupole Ion Trap Mass Spectrometer. I. Mass Spectrom. 1997, 32(6), 602-615.

5. Doroshenko, V. M.; Cotter, R. J. A New Method of Trapping Ions Produced by Matrix-Assisted Laser-Desorption Ionization in a Quadrupole Ion Trap. Rapid Commun. Mass Spectrom. 1993, 7(9), 822-827.

6. Fulford, J. E.; Hoa, D. N.; Hughes, R. J.; March, R. E.; Bonner, R. F.; Wong, G. J. Radio-Frequency Mass Selective Excitation and Resonant Ejection of Ions in a 3-Dimensional Quadrupole Ion Trap. J. Vac. Sci. Technol. 1980, 17(4), 829-835.

7. Lammert, S. A.; Cooks, R. G. Pulsed Axial Activation in the Ion Trap-a New Method for Performing Tandem Mass Spectroscopy (MS/MS) Rapid Commun. Mass Spectrom. 1992, 6(8), 528-530.

8. Murrell, J.; Despeyroux, D.; Lammert, S. A.; Stephenson, J. L.; Goeringer, D. E. "Fast Excitation" CID in a Quadrupole Ion Trap Mass Spectrometer. J. Am. Soc. Mass Spectrom. 2003, 14(7), 785-789.

9. Paradisi, C.; Traldi, P.; Vettori, U. Collisional Ion Activation in the Ion-Trap by a Three-Step Tickle Function. Rapid Commun. Mass Spectrom. 1993, 7(6), 440-445.

10. Cunningham, C.; Glish, G. L.; Burinsky, D. J. High Amplitude Short Time Excitation: A Method to Form and Detect Low Mass Product Ions in a Quadrupole Ion Trap Mass Spectrometer. J. Am. Soc. Mass Spectrom. 2006, 17(1), 81-84.

11. Jackson, G. P.; King, F. L.; Duckworth, D. C. Efficient Polyatomic Interference Reduction in Plasma-Source Mass Spectrometry via Collision Induced Dissociation. J. Anal. At. Spectrom. 2003, 18(9), 1026-1032.

12. Collin, O. L.; Beier, M.; Jackson, G. P. DCID of Peptides in a QIT Mass Spectrometer. Anal. Chem. 2007, 79(14), 5468-5473.

13. Jackson, G. P.; Hyland, J. J.; Laskay, Ü. A. Energetics and Efficiencies of Collision-Induced Dissociation Achieved During the Mass Acquisition Scan in a Quadrupole Ion Trap. Rapid Commun. Mass Spectrom. 2005, 19(23), 3555-3563.

14. Laskay, Ü. A.; Hyland, J. J.; Jackson, G. P. Dynamic Collision-Induced Dissociation (DCID) in a Quadrupole Ion Trap Using a Two-Frequency Excitation Waveform: I. Effects of Excitation Amplitude and Phase Angle. J. Am. Soc. Mass Spectrom. 2007, 18, 749-761.

15. Lu, Y. C.; King, F. L.; Duckworth, D. C. Electrochemically-Induced Reactions of Hexafluorophosphate Anions with Water in Negative Ion Electrospray Mass Spectrometry of Undiluted Ionic Liquids. J. Am. Soc. Mass Spectrom. 2006, 17(7), 939-944.

16. Harrison, A. G.; Lin, M. S. Energy-Dependence of the Fragmentation of the Normal-Butylbenzene Molecular ion. Int. J. Mass Spectrom. Ion Processes 1983, 51(2/3), 353-356.

17. March, R. E.; McMahon, A. W.; Londry, F. A.; Alfred, R. L.; Todd, J. F. J.; Vedel, F. Resonance Excitation of Ions Stored in a Quadrupole Ion Trap. 1. A Simulation study. Int. J. Mass Spectrom. Ion Processes 1989, 95(2), 119-156. 